\title{
Die moorgeologische Datierung des jüngeren Magdalénien in Deutschland
}

\author{
Von Hugo Gross, z. Zt. Bamberg
}

Das Problem der exakten geologischen Datierung des typischen westlichen und südlichen Magdalénien hat viele Prähistoriker und Geologen beschäftigt; man ist aber nicht über eine annähernde Einstufung in die relative glazialgeologische Zeitskala hinausgekommen. P. WOLDSTEDT (1929, S. 226 und 313) hat das Magdalénien in das Daniglazial (zu dem er auch den Langeland-Vorstoß und seine Abschmelzphase rechnete) vom Pommerschen Stadium an, in den Alpen vom Zürichstadium an, S. 292 aber genauer in die Zeit des LangelandVorstoßes (=Bühl-Stadium in den Alpen) gestellt. 1935 (S. 286) datiert derselbe Autor das Magdalénien ,in Norddeutschland etwa während des Pommerschen Stadiums, im alpinen Gebiet während des Zürich- Stadiums" und bemerkt, daß es in Norddeutschland noch keine Anhaltspunkte für die Datierung von Anfang und Ende des Magdalénien gebe. In der Schweiz ist nach H.-G. BANDI (1947, S. 44, 54) in der Hauptsache nur die jüngere Phase des Magdalénien (15 000 bis 10000 vor Chr.) vertreten, die in die Zeit einer Schneegrenzendepression von maximal $300 \mathrm{~m}$ fallen soll (diese Zahl wurde aber früher von PENCK und BRỦCKNER für das beträchtlich spätere Daun-Stadium angegeben!).

Bisher war noch für keine Station des typischen Magdalénien in West- und Süddeutschland eine genaue Einordnung in die geochronologische Zeitskala gelungen. Jetzt haben das aber die in den letzten Jahren erzielten Fortschritte der Moorgeologie ermöglicht.

Bald nach der Entdeckung von Kulturschichten der Hamburger Kultur am Grunde von Flachmooren bei Meiendorf und Stellmoor in Holstein durch A. RUST, dessen Grabungen auch eine exakte pollenanalytische Untersuchung dieser Moorprofile durch R. SCHÜTRUMPF ermöglichten, wurden im westlichen Norddeutschland (wie in Holland und Nordbelgien) andere steinzeitliche Fundplätze entdeckt, deren Kulturhinterlassenschaft nach H. SCHWABEDISSEN (1944) eine andere geschlossene Kulturgruppe repräsentiert, welche er „F e d e r messer-Gruppe“ genannt hat; dazu gehören z. B. die Fundplätze Grande (A. RUST 1943), Bornwisch (A. RUST 1948) und Rissen (H. SCHWABEDISSEN 1949) bei Hamburg. Im Gegensatz zu der Hamburger und Ahrensburger Kultur, deren Ursprung im Osten angenommen wird, weist die Federmesser-Gruppe nach dem Westen und Süden und ist jetzt von H. SCHWABEDISSEN (1949) auf Grund der Grabungsergebnisse in Rissen (1948) als s pätes Ma g d a lén ien erkannt worden, das älter ist als die Ahrensburger Stufe (der jüngeren DryasZeit), da im Dünenprofil von Rissen die Magdalénien-Kulturschicht (mit Spuren einer Sommerhütte) 1-1,20 m unter einer Kulturschicht der Ahrensburger Stufe festgestellt wurde. An der Bornwisch bei Ahrensburg fand A. RUST (1948) eine Kulturschicht (mit Winterbehausung) der Federmesser-Gruppe und in der Alleröd-Schicht des nahe gelegenen Teiches 2 Kulturschichten derselben Kultur. H. SCHWABEDISSEN (1949) vermutet richtig, daß der Aufenthalt der Magdalénien-Leute in der Hamburger Gegend und damit im nordwestdeutschen Flachland „auf ein wärmeres Klima etwa der Alleröd-Zeit“ hinweise und hebt hervor, daß sich hier „zum ersten Male die Möglichkeit einer Parallelisierung des westlichen Magdalénien mit einer der Kulturgruppen des Nordens" bietet.

Daher ist jetzt die Frage der genauen zeitlichen Einordnung auch des westlichen und südlichen Magdalénien aktuell. Bisher gibt es noch kein Pollendia- 
gramm, das eine solche Kulturschicht erfaßt hat. Ich habe aber bereits 1946 einige Fachleute auf eine Datierungsmöglichkeit hingewiesen, die ich nach dem Verlust meiner wissenschaftlichen Akten infolge des letzten Krieges erst jetzt verfolgen konnte.

Im Neuwieder Becken am Rhein lag am Martinsberg bei Ander $\mathrm{nach}$ eine 1883 von H. SCHAAFFHAUSEN (1888) ausgegrabene Magdalénien-Station unter einer ca. $4 \mathrm{~m}$ mächtigen Bimssandschicht mit Tuffsanddecke, die vulkanischen Ausbrüchen der Laacher Gegend entstammen (A. GÜNTHER 1924, S. 50 ff.). Knochen und Geräte aus Rengeweih und Knochen nebst über 300 Artefakten aus Stein lagen nach J. ANDREE (1939, S. 545) in und a u dem verlehmten Löß, der einen älteren Lavastrom bedeckte und die bis ca. $1 \mathrm{~m}$ tiefen Lücken, zwischen den Lavablöcken ausfüllte, nach H. SCHAAFFHAUSEN (1888, S. 8, S. 22 ff.) aber alle in dieser Lehmschicht von ihrer Oberfläche an. Dieser seit Beginn der Besiedelung eingewehte oder eingeschwemmte Löß ist verlehmt und etwas humos, zeigt also deutliche Spuren chemischer Verwitterung. Die Knochen und Artefakte aus Rengeweih und Knochen waren zum Teil durch die Verwitterung angegriffen; leider sind die Funde damals nicht schichtenweise getrennt worden. Es ist anzunehmen, daß sie vor der Bedeckung mit Bimssand nicht lange in diesem Boden gelegen haben können, daß also zwischen der letzten Besiedelung und dem Bimssandauswurf kein nennenswerter zeitlicher Abstand liegen kann; andernfalls wäre der Wohnplatz vor dem Bimssandauswurf höchst wahrscheinlich mit Schwemmlehm vom Martinsberg oder mit einer Humusschicht bedeckt worden. Nach R. R. SCHMIDT (Prähist. Zeitschr. 1, 1909, S. 343-346) war diese Station vom Hoch- bis zum Spät-Magdalénien bewohnt; J. ANDREE (1939, S. 545-546) meinte, daß hier eine echte Spätstufe der jüngeren Klingenkultur nicht vorzuliegen scheine, da die feine Mikrolithik aus dem Ende dieser Kultur fehle.

Die Fauna der Fundstelle weist nach H. SCHAAFFHAUSEN (1888, S. 30) auf (nach der Häufigkeit geordnet): Equus caballus fossilis, Rangifer tarandus, Bos primigenius, Vulpes lagopus, Cervus elaphus, Arvicola amphibius, Mustela vulgaris, Mus musculus, Lagopus albus, Canis lupus, Lepus variabilis, Felis lynx, Sciurus vulgaris, Talpa europaea, Strix (brachyotus?), Anas (boschas?), Grus cinerea oder Cygnus musicus?, Tetrao bonasia, ferner 2 Schneidezähne von einem 2jährigen Kind und 7 menschliche Rippenstücke (davon 2 an 1 bzw. 2 Enden rundlich zugeschliffen). Die großen dulivialen Säuger Mammut, Wollhaarnashorn und Moschusochs sind verschwunden, auch der Halsbandlemming und seine Artgenossen fehlen; Rothirsch, Luchs und Eichhörnchen, die an Waldwuchs gewöhnt sind, zeigen, wie schon H. HECK (1925) hervorhob, bereits den Beginn eines wärmer werdenden Klimas an (der Rothirsch trat in Holstein und Dänemark erstmalig in der Alleröd-Zeit auf, wie mir Herr Dr. A. RUST brieflich mitteilte.) Auf größere Wärme und stärkereNiederschläge deutet vor allem die Verlehmung des (jüngeren) Lößes hin, ferner das Vorkommen von Baumresten in der untersten Schicht des Bimssandes an anderen Stellen des Neuwieder Beckens, aus denen man "höchstens auf einen dürftigen und krüppelhaften Baumwuchs“ schließen kann (A. GÜNTHER 1924, S. 55). Im Brohl- und Condertal sind in den Trass- und Tuffablagerungen, deren Zeitstellung und Herkunft dieselben wie beim Bimssand sind, spärliche Tier- und zahlreiche Pflanzenreste gefunden worden. Die älteren botanischen Angaben (von WIRTGEN und JAKOBS), aber auch die neueren von SCHLICKUIM und R. POTONIE sind, wie aus der sehr sorgfältigen Untersuchung der Pflanzenreste aus den vulkanischen Tuffen der Eifel von R. KRÄUSEL und H. WEYLAND (1942) hervorgeht, für die Datierung 
des großen Bimssandauswurfs unbrauchbar, da viele Artbestimmungen falsch waren und ein Teil der Pflanzenreste aus interglazialen Tuffen stammte.

Aus seinen zahlreichen stratigraphischen Untersuchungen zieht A. GÜNTHER (1924, S. 57,58) den Schluß: „Im großen und ganzen wird man nur mit einem einmaligen, wenn auch mit kurzen Unterbrechungen und stellenweise veränderter Richtung andauernden Bimssandauswurf mit der gleichzeitigen Tuff- und Trassablagerung rechnen müssen, dem dann nach verhältnismäßig kurzer Zeitdauer und als Schluß der ganzen Vulkantätigkeit der graue Tuffsandauswurf folgte“, denn die Oberfläche des weißen Bimssandes zeigt „kaum die Spur einer Verwitterung oder gar Verlehmung" (oder Humusbildung). Dieselbe Ansicht wird von W. AHRENS (W. AHRENS und K. STEINBERG 1943, S. 26) vertreten: „Einer Serie rasch aufeinanderfolgender Ausbrüche verdanken die weißen Bimssteine, die das Neuwieder Becken erfüllen, ihre Entstehung. Unwesentlich jünger sind die grauen Trachyttuffe; sie entstammen nah benachbarten Ausbruchsstellen im jetzigen Kessel des Laacher Sees“.

Die Bimssandüberschüttung erfolgte nach A. GÜNTHER (1924, S. 51) erst $\mathrm{n} \mathrm{a} \mathrm{ch}$ der Bildung der diluvialen Niederterrasse, als diese schon eine verlehmte Oberfläche und einen wenn auch dürftigen und krüppelhaften Baumwuchs trug; auf Grund der ihm bekannten botanischen Angaben stellte er den Bimssandauswurf an das Ende des Diluviums in den „Übergang des Daun-Stadiums zum Alluvium (Azilien-Tardenoisien)“, zumal der Bimssand nur von einer Tuffsandschicht und Dammerde bedeckt ist. R. KRÄUSEL und H. WEYLAND (1942 S. 34-39) halten den Bimsstein-Ausbruch für postglazial, er dürfte nach ihrer Ansicht ,wahrscheinlich.schon in den Ausgang der Kiefern-Birkenzeit zu verlegen sein" (d.h. in den Übergang von der Yoldia- zur Ancylus-Zeit, um 7500 vor Chr.); die grauen Trachyt-Tuffe und grauen Tuffsande dürften nach ihrer Ansicht jünger sein „wofür das Auftreten von Carpinus betulus spricht“. Dann müßte aber der Tuffsandausbruch (im Widerspruch zum stratigraphischen Befund) mehrere tausend Jahre jünger sein als der Bimssandauswurf, denn Carpinus betulus ist erst in der zweiten Hälfte der postglazialen Wärmezeit eingewandert. Von den grauen Tuffen sagen R. KRÄUSEL und H. WEYLAND (1942 S. 35): „Fraglich bleibt, ob sie nicht etwa in wesentlich späterer Zeit umgelagert sein könnten, d. h. also, ob die eingeschlossenen Pflanzenreste nicht viel jünger sein könnten als die Tuffe“. Diese Möglichkeit wird zur Gewißheit, wann man bedenkt, daß Blätter von der Weißbuche, aber nicht von der sehr viel häufigeren Rotbuche und Eiche gefunden worden sind; auch die Blätter des Bergahorns (Acer pseudoplatanus) dürften in umgelagertem Tuffsand gelegen haben; das schlecht erhaltene „Bergahorn“-Blatt aus den weiBen Bimssteintuffen des Brohltals stammt möglicherweise von Viburnum opulus, wie auch Herr Prof. Dr. FIRBAS (briefl. Mitteil.) meint.

Daß die oben erwähnten Datierungen nicht ganz richtig sind, geht aus dem Profil vom Wildweiberhausfelsen im Westerwald hervor (H. BEHLEN 1905, H. HECK 1925, S. 111), wo weißer Bimssand von derselben Zeitstellung und Herkunft wie im Neuwieder Becken (auf Felsabwitterung und Lehm mit zum Teil arktischer und subarktischer Fauna: Myodes torquatus, Lagopus albus, L. alpinus, Rangifer tarandus, Vulpes lagopus) von humosem Lehm überlagert wird, der unten Lagomys pusillus, Lagopus albus und Rangifer tarandus enthält. Diese Fauna des Hangenden der vulkanischen Schichten spricht gegen postglaziales Alter.

Da der Bimssandauswurf aus dem Laacher Gebiet nach A. GÜNTHER (1924, S. 51) einem einheitlichen Vulkanausbruch entstammt, bei dem die Periode des 
weißen Bimssandes von der Periode des ihn überlagernden grauen Tuffsandes nur durch einen sehr kurzen Zeitraum getrennt sein kann und da dieser Auswurf von außerordentlicher Ausdehnung war (bis 1924 ostwärts bis Marburg nachgewiesen), kann er jetzt exakt moorgeologisch datiert werden: der Tuffsand ist nämlich ostwärts über Göttingen hinaus verweht und als d ünne Einlagerung mitten in Allerödschichten im Eichsfeld (W. AHRENS und K. STEINBERG 1943) östlich und in einem von P. W. THOMSON bei Wallensen nördlich von Göttingen entdeckten Mooraufschluß von F. FIRBAS (laut briefl. Mitteil.) festgestellt worden, und zwar als einzige Einlagerung vulkanischer Herkunft. Der Vulkanausbruch, der die Magdalénien-Station Andernach (und bei Weißenturm sogar einen Menschen nach A. GÜNTHER 1924, S. 53 ) verschüttet hat, ist also allerödzeitlich (zwischen 9000 und 10000 vor Chr.).

Die Fauna der Fundstelle (vor allem das Vorkommen des Rothirsches) und das Fehlen einer Schwemmlehmdecke oder Humusschicht auf der Fundschicht sprechen dafür, daß die Magdalénien-Station noch in der frühen Alleröd-Zeit (sehr wahrscheinlich bis zum Bimssandauswurf) bewohnt war. Der Beginn der Besiedlung, d.h. die Zeitstellung des Hoch-Magdalénien von Andernach, kann schon wegen der damals unvollkommenen Grabungstechnik an dieser Station nicht so gut wie das Ende ermittelt werden; er fällt offenbar in das Ende der Lößablagerungszeit, d.h. in den Endabschnitt der baumlosen Tundrenzeit. Dafür sprechen auch die Befunde von der Magdalénien-Station an der Schussenquelle. Die Moostorfschicht, die hier die Magdalénienfunde enthielt, ist nach dem Ergebnis der pollenanalytischen Untersuchung in der baumlosen Tundrenzeit abgelagert worden (F. FIRBAS 1935); nach R. R. SCHMIDT liegt hier Mittel-Magdalénien vor (vielleicht Übergang zum Spät-Magdalénien), nach G. RIEK Hoch- bis Spät-Magdalénien, nach H.-G. BANDI (1947, S. 21) sogar Magdalénien 6; aber gegen die Datierung „End-Magdalénien“ spricht schon das Fehlen des Rothirsches. Im Hinblick auf die Zeitstellung der Magdalénien-Station von Andernach (zufolge der Typologie und den geologischen Befunden) kann die Fundschicht an der Schussenquelle nur aus dem Schlu Babschnitt der baumlosen Tundrenzeit stammen, ebenso wie die Fundschichten der Hamburger Kultur in Meiendorf und Stellmoor nach den Pollendiagrammen von $\mathrm{R}$. SCHÜTRUMPF. Da der Schlußabschnitt der baumlosen Tundrenphase der älteren Dryas-Zeit nach meinen Untersuchungen im nördlichen Ostpreußen f $\mathrm{r} \ddot{\mathrm{u}} \mathrm{h}-\mathrm{g}$ ot i g la zi a 1 ist, können m. E. die Fundschichten der Hamburger Kultur auch nur früh-gotiglazial sein, wie ich es schon vor Jahren behauptet habe ${ }^{\mathbf{1}}$ ). Von „mittel-würmglazialem" Alter kann zweifellos keine Rede sein, ebenso wenig von geringer Entfernung des Inlandeisrandes von diesen Renntierjägerlagern ${ }^{2}$ ) (vergl. die Angabe der maximalen Schneegrenzen-Depression in den Alpen im jüngeren Magdalénien bei H.-G. BANDI 1947 S. 44 und seine Karte). Es kommt natürlich nicht darauf an, ob eine solche spätglaziale Kulturschicht ein paar tausend Jahre älter oder jünger als eine Kulturschicht anderwärts ist, sondern auf eine möglichst

1) A. BOHMERS (im Nieuwedrentsche Volksalmanak 1948 S. 103) stellt die Hamburger Kultur I auch in die Zeit zwischen 14000 und 12000 vor Chr.

2) Der gebänderte, also warwig aussehende Sand im Liegenden dieser Kulturschichten ist kein Beweis dafür, da er Tundra-Pollen enthält, was bisher noch für keine einwandfreie Eisstauseebildung im südlichen Ostsee-Gebiet nachgewiesen werden konnte, und da derartige gebänderte Schichten (mit anderer Fossilführung natürlich) bisweilen noch in der jüngeren Dryas-Zeit abgelagert worden sind (z. B. in Alleröd in Dänemark, mehrfach in Ostpreußen, Lettland und Estland). 
zuverlässige glazialgeologische Einstufung. Denn auch diese Kulturschichten der Hamburger Kultur legen offenbar einen wichtigen stratigraphischen und geochronologischen Leithorizont in Norddeutschland fest, den Beginn des Gotiglazials (15 000 vor Chr. nach der letzten Datierung von G. DE GEER), in dessen frühe Abschmelzphase (etwa zwischen 14000 und 12000 vor Chr.) diese Kulturschichten aller Wahrscheinlichkeit nach zu stellen sind. D a s f üh e G otig la zi a 1 ist offenbar eine erste beträchtliche Wärmeschwankung von ähnlicher Bedeutung wie die Alleröd-Schwankung und die finiglaziale Klimabesserung; dafür spricht die geradezu explosionsartige Massenausbreitung der Anodonten, die Tiere eines gemäßigten Klimas sind, seit dem Schlußabschnitt der baumlosen Tundrenzeit im südlichen Ostseegebiet (in Stellmoor unmittelbar über der Kulturschicht der Hamburger Kultur nach A. RUST 1943, S. 46, 47), die Einwanderung und Ausbreitung der ersten Waldbäume sowie die Einwanderung der paläolithischen Renntierjäger in den südlichen Nord- und Ostsee-Raum. H. GAMS läßt mit Recht mit der gotiglazialen Anfangsmoräne (Langeland-Vorstoß) das Spätglazial beginnen. Die genaue glazialgeologische Einstufung der Magdalénien-Station Andernach gestattet die Schlußfolgerung, daß die Einwanderung der norddeutschen Renntierjäger in der zweiten Hälfte des (westlichen) Magdalénien erfolgte.

Es muß also jetzt möglich sein, in Nordwestdeutschland moorgeologisch das Ende des Magdalénien zu datieren. Aber auch in Süd-Deutschland besteht die Möglichkeit, das jüngere Magdalénien moorgeologisch zu datieren, da dort (und in den Alpen) verschiedene Pollendiagramme bis in die ältere Dryas-Zeit (=Abschmelzphase des Bühlstadiums) zurückreichen. An der Schussenquelle war das Hangende des Moostorfs mit den Magdalénien-Funden Kalktuff und darüber Torf.

Aber auch in Frankreich ist die Aussicht vorhanden, daß wenigstens das Ende des dortigen Magdalénien moorgeologisch erfaßt werden kann; G. und C. DUBOIS (Comptes rendus de la Soc. géol. de France V, S. 46-48, und VI, S. 61-63, 1944) fanden im französischen Zentralplateau im Massiv von Cantal in Hö̉hen von 950 und $1100 \mathrm{~m} 2$ Schichten, die sicher allerödzeitlich sind, wie die Verfasser annehmen (neben Birken und Kiefern in den tieferen Lagen auch kräftige Ausbreitung von Eichen in dieser Zeit). Eine Allerödschicht mit Pollen von Querus, Corylus und Alnus findet sich vielleicht auch in dem von denselben Autoren (Ann. Soc. géol. Nord, Lille, 65, S. 151-158, 1945) untersuchten Moor von Esar am Ostfluß der Argonnen ${ }^{3}$ ). „Aus einigen Torfmooren an der Mündung der Somme hat sich BOUCHER DE PERTHES rohbehauene Steinwerkzeuge und Reste vom irischen Riesenhirsch geholt" ${ }^{4}$ ). Diese Artefakte dürften in den "Antiquités celtiques et antédiluviennes" von BOUCHER DE PERTHES, die mir nicht zugänglich sind, abgebildet und für den Prähistoriker archäologisch (Magdalénien?) datierbar sein. Der Riesenhirsch ist in Nordwesteuropa, soweit bekannt, im Spätglazial ausgestorben; eine Torfschicht mit Riesenhirschresten in der Nähe der Somme-Mündung dürfte also allerödzeitlich sein, so daß die erwähnten Artefakte mit großer Wahrscheinlichkeit auch geologisch datiert werden können, wenn sie in derselben Moorschicht wie die Riesenhirschreste gefunden worden sind.

Der Beginn des Magdalénien wird sich so nicht ermitteln lassen, da die (kontinuierlichen) Pollendiagramme, nach der überall in Norddeutschland festge-

3) Nach F. FIRBAS „Systematische und genetische Pflanzengeographie“ (Fortschritte der Botanik 12, 1949). Ich bin Herrn Prof. FIRBAS für die Zusendung der Korrekturbogen dieses Beitrags sehr zu Dank verpflichtet.

${ }^{5}$ ) Nach N. JOLY „Der Mensch vor der Zeit der Metalle“, Leipzig 1880, S. 112. 
stellten geringen Schichtenmächtigkeit der baumlosen Tundrenzeit zu urteilen, offenbar nicht über das Gotiglazial zurückreichen; bis dahin kann die pollenanalytisch erfaßbare Sedimentation nur durch Erdfließen verhindert worden sein. $\mathrm{Da}$ die Klimadepression zur Zeit des mittelschwedischen Eisrandgürtels (also in der jüngeren Dryas-Zeit) bis in die Alpen hinein in den Schichtenfolgen und Pollendiagrammen so deutlich erkennbar ist, müßte der Langeland-Vorstoß (= Bildung der gotiglazialen Anfangsmoräne) stratigraphisch und pollenanalytisch erst recht deutlich nachweisbar sein (was aber nicht der Fall ist), wenn in Deutschland die Pollendiagramme über das Gotglazial hinaus zurückreichen würden.

Das Verdienst, die Möglichkeit entdeckt und bewiesen zu haben, mit Hilfe vulkanischer Schichten exakte geologische Datierungen auszuführen, gebührt V. AUER (vergl. seine zusammenfassende Arbeit von 1948).

$\mathrm{Schriftenverzeichnis}$

AHRENS, W. und STEINBERG, K. (1943): Jungdiluvialer Tuff im Eichsfeld. Ber. Reichsamt f. Bodenforschung Jahrg. 1943.

ANDREE, J. (1939) Der eiszeitliche Mensch in Deutschland. - Stuttgart.

AUER, V. (1948) Las capas volcánicas como nuevo método de cronologia postglazial en Fuegopatagonia.- Gaea (Buenos Aires) 8, S. 311-336.

BANDI, H.-G. (1947) Die Schweiz in der Rentierzeit. Frauenfeld.

BEHLEN, H. (1905) Das Alter und die Lagerung des Westerwälder Bimssandes. Jahrb. d. Nassauischen Vereins f. Naturk. 58, S. 44-51.

FIRBAS, F. (1935) Die Vegetationsentwicklung des mitteleuropäischen Spätglazials. Bibliotheca Botanica H. 112.

GÜNTHER, A. (1924) Vulkantätigkeit und Eiszeit im östlichen Eifelvorland zwischen Mosel und Vinxtbach. - „Die Eiszeit“ 1. S. 46-59.

HECK, H. (1925) Das rheinische Paläolithikum. - „Die Eiszeit“ 2, S. 96-111.

KRÅUSEL, R. und WEYLAND, H. (1942) Tertiäre und quartäre Pflanzenreste aus den vulkanischen Tuffen der Eifel. - Abh. senckenberg. naturf. Ges. Nr. 463, S. $1-62$.

RUST, A. (1943) Die alt- und mittelsteinzeitlichen Funde von Stellmoor. - Neumünster. - (1948) Jungpaläolithische Wohnanlagen bei Hamburg. — „Hammaburg" 1 , S. 33-38.

SCHAAFFHAUSEN, H. (1888) Die vorgeschichtliche Ansiedlung in Andernach. Jahrbücher d. Ver. v. Altertumsfreunden im Rheinlande 86, S. 1-41, Bonn.

SCHWABEDISSEN, H. (1944) Die mittlere Steinzeit im westlichen Norddeutschland. Neumünster. - (1949) Hamburg-Rissen, ein wichtiger Fundplatz der frühen Menschheitsgeschichte. - „Hammaburg“ 2, S. 81-90.

WOLDSTEDT, P. (1929) Das Eiszeitalter. - Stuttgart. - (1935) Die Beziehungen zwischen den nordischen Vereisungen und den paläolithischen Stationen von Nord- und Mitteldeutschland. - „Mannus“ 27, S. 275-287.

\section{Australopithecus oder Plesianthropus oder Paranthropus?}

\section{Von Hans Weinert, Kiel}

Als 1924 bei Taungs im Betschuanaland der Kinderschädel gefunden wurde, dem DART die Bezeichnung Australopithecus africanus gab, da zweifelte niemand daran, daß diese Genus-Bezeichnung richtig sei. Denn trotz aller menschlichen Merkmale im Verhältnis von Gehirn- zu Gesichtsschädel und vor allen Dingen trotz des Zahnbogens und Gebisses war die absolute Größe und auch die Form des Schädels so schimpansenähnlich, daß sicher ein Anthropoide und kein Hominide vorlag. Zwar fiel die Größe der ersten Dauermolaren auf, sodaß manche 